\title{
Perfil clínico-epidemiológico de pessoas acometidas por úlceras neurotróficas decorrentes de hanseníase
}

\author{
Clinical-epidemiological profile of people affected by neurotrophic ulcers resulting from leprosy \\ Perfil clínico-epidemiológico de las personas afectadas por úlceras neurotróficas derivadas de la \\ lepra
}

Recebido: 31/08/2021 | Revisado: 10/09/2021 | Aceito: 18/09/2021 | Publicado: 19/09/2021

Camila Isnaide Pimentel Pinheiro

ORCID: https://orcid.org/0000-0001-5059-5725

Universidade Estadual do Piauí, Brasil

E-mail: isnayd@gmail.com

Isabel Cristina Cavalcante Carvalho Moreira

ORCID: https://orcid.org/0000-0003-2717-2534

Universidade Estadual do Piauí, Brasil

E-mail: isabelccavalcante@hotmail.com

Silvia Cristina Nunez

ORCID: https://orcid.org/0000-0003-2101-8333

Universidade Brasil, Brasil

E-mail: silvia.nunez@universidadebrasil.edu.br

Talita de Brito Silva

ORCID: https://orcid.org/0000-0001-9710-7012

Universidade Estadual do Piauí, Brasil

E-mail: talitadebritosilva@yahoo.com.br

Mariana Soares Pereira

ORCID: https://orcid.org/0000-0001-6948-0266

Universidade Estadual do Piauí, Brasil

E-mail: mariana.s2110@gmail.com

Davi Pinheiro Campelo

ORCID: https://orcid.org/0000-0002-4668-1062

Instituto Federal do Piauí, Brasil

E-mail: davi123mgil@gmail.com

Samira Rego Martins de Deus Leal

ORCID: https://orcid.org/0000-0003-3438-5992

Universidade Estadual do Piauí, Brasil

E-mail: samirarego@ccs.uespi.br

Renata Celestino Nunes

ORCID: https://orcid.org/0000-0002-8798-6970 Universidade Estadual do Piauí, Brasil E-mail: renatacelestino18@gmail.com

Verônica Elis Araújo Rezende

ORCID: https://orcid.org/0000-0001-9076-3375

Centro Integrado de Saúde Lineu Araújo, Brasil E-mail: veronicaelisrezende@yahoo.br

Sandra Marina Gonçalves Bezerra ORCID: https://orcid.org/0000-0003-3890-5887 Universidade Estadual do Piauí, Brasil E-mail: sandramarina20@ hotmail.com

Antonio Luis Martins Maia Filho

ORCID: https://orcid.org/0000-0001-6184-8003 Universidade Estadual do Piauí, Brasil

E-mail: almmaiaf@ccs.uespi.br

\begin{abstract}
Resumo
O objetivo deste estudo foi identificar o perfil epidemiológico de pessoas com úlceras neurotróficas decorrentes de hanseníase, por meio de um estudo clínico com abordagem quantitativa, caracterizado pelo uso da quantificação na coleta de dados e consequente tratamento por técnicas estatísticas. Sendo realizada nas dependências do Centro Maria Imaculada, referência para hanseníase no município de Teresina, região Nordeste do Brasil. A média de idade foi de 54,4 anos, $70 \%$ participantes eram do sexo masculino, 60,0\% possuíam menos de oito anos de estudo formal, a renda de $60 \%$ da renda mensal era constituída por aposentadoria e $50 \%$ recebiam dois salários-mínimos mensalmente, a raça de $50 \%$ era parda e o Piauí foi o estado de residência de todos e 70,0\% dos indivíduos tinha a HAS como comorbidades associada. O tempo médio de evolução da úlcera neurotrófica foi de 101,8 meses e 30,0\% das lesões
\end{abstract}


eram no hálux. Estes achados refletem o perfil de indivíduos com diversas limitações e situações socioeconômicas desfavoráveis, que reforçam a associação entre pobreza e hanseníase gerando necessidade de capacitação contínua dos profissionais de saúde que prestam assistência aos pacientes com hanseníase, em todos os níveis de atenção à saúde.

Palavras-chave: Perfil epidemiológico; Hanseníase; Lesões; Saúde.

\begin{abstract}
The aim of this study was to identify the epidemiological profile of people with neurotrophic ulcers resulting from leprosy, through a clinical study with a quantitative approach, characterized by the use of quantification in data collection and subsequent treatment by statistical techniques. It was carried out on the premises of the Centro Maria Imaculada, a reference center for leprosy in the municipality of Teresina, Northeast region of Brazil. The average age was 54.4 years, $70 \%$ of the participants were male, the majority, $60.0 \%$ had less than eight years of formal education, $60 \%$ of the income consisted of retirement and 50\% received two salaries -minimum monthly, 50\% of the race was mixed race and Piauí was the state of residence of all and $70.0 \%$ of the individuals had SAH as associated comorbidities. The average time of evolution of the neurotrophic ulcer, in months, was 101.8. 30.0\% of the lesions were in the hallux. These findings reflect the profile of individuals with various limitations and unfavorable socioeconomic situations, which reinforce the association between poverty and leprosy, generating the need for continuous training of health professionals who provide care to patients with leprosy, at all levels of health care.
\end{abstract}

Keywords: Epidemiological profile; Leprosy; Injuries; Health.

\title{
Resumen
}

El objetivo de este estudio fue identificar el perfil epidemiológico de las personas con úlceras neurotróficas derivadas de la lepra, mediante un estudio clínico con enfoque cuantitativo, caracterizado por el uso de la cuantificación en la recolección de datos y posterior tratamiento por técnicas estadísticas. Se llevó a cabo en las instalaciones del Centro María Imaculada, centro de referencia en lepra en el municipio de Teresina, región Nordeste de Brasil. La edad promedio fue de 54.4 años, el $70 \%$ de los participantes eran hombres, la mayoría, el $60.0 \%$ tenía menos de ocho años de educación formal, el $60 \%$ de los ingresos consistía en jubilación y el 50\% recibía dos salarios -minimo mensual, $50 \%$ de la raza era mestiza y Piauí era el estado de residencia de todos y el 70,0\% de los individuos tenían HSA como comorbilidades asociadas. El tiempo medio de evolución de la úlcera neurotrófica, en meses, fue de 101,8. El 30,0\% de las lesiones estaban en el hallux. Estos hallazgos reflejan el perfil de individuos con diversas limitaciones y situaciones socioeconómicas desfavorables, que refuerzan la asociación entre pobreza y lepra, generando la necesidad de una formación continua de los profesionales de la salud que brindan atención a los pacientes con lepra, en todos los niveles de la atención de salud.

Palabras clave: Perfil epidemiológico; Lepra; Lesiones; Salud.

\section{Introdução}

A hanseníase ou doença de Hansen é caracteriza-se por ser uma moléstia infectocontagiosa de curso crônico e início insidioso, granulomatosa, de baixa patogenicidade e curável causada pelo agente etiológico Mycobacterium leprae (M. leprae) (Almeida, 2016; Alves et al., 2017; Almeida \& Milan, 2020). Após a contaminação, a micobactéria se instala na pele e células de Schwann e inicia seu processo de multiplicação, apresentando lesões dermatológicas e causando danos neurológicos com perdas axonais importantes, resultando em alterações na sensibilidade térmica, dolorosa e tátil e até perda da função (Batista et al., 2019; Arruda et al., 2020).

Integrante do grupo de doenças negligenciadas, a hanseníase está inserida em um contexto de condições de pobreza, revelando a negligência sofrida por alguns grupos da sociedade, e indicando divergentes intensidades de vulnerabilidade, em um âmbito que perpassa o econômico, atingindo também o individual e social, apresentando-a como problema de saúde pública entre alguns países em desenvolvimento (Chaves et al., 2015; Almeida, 2016; Araújo \& Araújo, 2017).

Neste cenário, em 2016, no Brasil, a taxa de detecção de casos foi de 12,2/100 mil habitantes, classificando-o como hiperendêmico (Pêgo et al., 2020). Desta forma, o Brasil ocupa o segundo lugar na relação de países com maior número de casos no mundo, tornando-se alvo de ações prioritárias pelo Ministério da Saúde (MS), visando à detecção precoce de casos e o exame de contatos, desenvolvimento de pesquisas básicas, tratamento imediato com esquema de PQT e enfrentamento do estigma (OMS, 2016). Os maiores coeficientes de prevalência da hanseníase têm sido observados em regiões mais pobres do Brasil, a saber: Norte, Nordeste e Centro-oeste, com padrões que refletem endemicidade para a doença em locais com 
expressiva desigualdade socioeconômica. Por consequência, a região Nordeste apresenta alta endemicidade e a capital do estado do Piauí, Teresina, foi classificada como a segunda capital do país mais hiperendêmica (Araújo, 2015; Andrade et al., 2016; Costa et al., 2016; Schneider \& Freitas, 2018; Araújo et al., 2020).

Se a hanseníase afetasse tão exclusivamente pele, não teria o potencial de interferir na saúde pública como o faz, porém o acometimento dos nervos torna seu controle de extrema necessidade. Dentre as consequências da redução ou ausência de sensibilidade, há maior susceptibilidade para o desenvolvimento de úlceras neurotróficas, principalmente na região plantar, resultante de lesões repetidas na região dos MMII e em graus diferentes de profundidade e gravidade, podendo levar a amputação de dígitos ou plantar (Batista et al., 2019; Chagas et al., 2019). Além disso, atinge qualquer faixa etária ou sexo, embora crianças adoeçam mais quando há maior endemicidade e homens mais que mulheres, afetando o indivíduo em sua vida social e laboral (Brasil, 2018; Brandão et al., 2020). Apesar de todo o empenho, em 2018, o Brasil apresentava o valor de 10,08\% de pacientes diagnosticados com grau 2 de incapacidade causada pela hanseníase e o Piauí, 20,16\%, resultado maior que a média nacional (Brasil, 2018; Ribeiro et al., 2018).

No que tange à Qualidade de Vida $(\mathrm{QV})$, a hanseníase afeta-a de diversas formas, seja em relação à saúde como as relações interpessoais. As alterações das funções, deformidades, úlceras neurotróficas e incapacidade física produzem uma série de mudanças na vida dos indivíduos acometidos por elas e, por consequência, na vida de seus ciclos sociais. As úlceras crônicas causadas pela hanseníase demonstram um impacto negativo sobre a QV dos indivíduos, afetando-os em diferentes níveis de suas rotinas (Oliveira, 2014).

O presente trabalho tem como objetivo o estudo e identificação do perfil epidemiológico de pessoas com úlceras neurotróficas decorrentes de hanseníase, no munícipio de Teresina no Piauí.

\section{Metodologia}

Trata-se de um estudo clínico com abordagem quantitativa, caracterizado pelo uso da quantificação na coleta de dados e consequente tratamento por técnicas estatísticas, compilando os dados de forma numérica e produzindo tabulações em gráficos e/ou tabelas (Campana et al, 2001). Foi desenvolvido nas dependências do Centro Maria Imaculada referência para hanseníase no município de Teresina, região Nordeste do Brasil.

A coleta de dados se deu durante a permanência dos pesquisadores no centro de referência, entre os meses de janeiro de 2020 a fevereiro de 2021, através de uma entrevista semiestruturada com aspectos epidemiológicos. Os dados foram analisados usando Statistical Package for the Social Sciences (SSP, Chicago, ILK, EUA), versão 20.0. O estudo foi desenvolvido após submissão e aprovação pelo Comitê de Ética e Pesquisa da Universidade Brasil sob protocolo n ${ }^{\circ}$ 3.706.331, mediante assinatura de termo de consentimento livre e esclarecido por parte dos voluntários.

\section{Resultados}

O estudo clínico-epidemiológico resultou na avaliação de 10 participantes com hanseníase e o perfil sociodemográfico é apresentado na Tabela 1. A média de idade foi de 54,4 anos, variando entre 35 e 68 anos. Dentre os sujeitos, $70 \%$ eram do sexo masculino.

Quanto ao estado civil 50\% dos participantes eram solteiros, a raça de 50\% era parda e o Piaú foi o estado de residência de todos. No tocante à escolaridade, a maioria (60\%) possuía menos de oito anos de estudo formal. A renda foi majoritariamente da aposentadoria $(60 \%)$ e $50 \%$ recebiam 2 salários-mínimos mensalmente. Quanto à doença associada, $70,0 \%$ dos indivíduos relataram a hipertensão arterial sistêmica (HAS) como comorbidade associada. 
Research, Society and Development, v. 10, n. 12, e235101220090, 2021

(CC BY 4.0) | ISSN 2525-3409 | DOI: http://dx.doi.org/10.33448/rsd-v10i12.20090

Tabela 1: Análise descritiva do perfil sociodemográfico dos participantes com hanseníase (n=10) em Teresina - Piauí.

\begin{tabular}{llc}
\hline & \multicolumn{2}{l}{ Hanseníase } \\
\hline Sexo & $\mathrm{n}$ & $\%$ \\
\hline Feminino (F) & 3 & 30,0 \\
Masculino (M) & 7 & 70,0 \\
Estado civil & & \\
Solteiro & 5 & 50,0 \\
Casado & 4 & 40,0 \\
Viúvo & 1 & 10,0 \\
Raça/Cor & & \\
Branca & & 10,0 \\
Parda & 1 & 50,0 \\
Negra & 5 & 50,0 \\
Amarela & 3 & 30,0 \\
\hline
\end{tabular}

Localização

\begin{tabular}{llc} 
Piauí & 10 & 100,0 \\
Escolaridade & & \\
$<8$ anos & 6 & 60,0 \\
\hline Entre 8 e 11 anos & 4 & 40,0 \\
Renda & & \\
Um salário mínimo & 4 & 40,0 \\
\hline Dois salários mínimos & 5 & 50,0 \\
\hline Três salários mínimos & 1 & 10,0 \\
Profissão & & \\
\hline Aposentado & 6 & 60,0 \\
\hline Funcionário público & 1 & 10,0 \\
Desempregado & 1 & 10,0 \\
Gerente de loja & 1 & 10,0 \\
Vendedor & 1 & 10,0 \\
Doença associada & & \\
\hline Hipertensão Arterial Sistêmica & & 70,0 \\
\hline Não & 7 & 30,0 \\
\hline Total & $\mathbf{1 0}$ & $\mathbf{1 0 0 , 0}$ \\
\hline
\end{tabular}

Fonte: Autores (2021).

O tempo médio de evolução da úlcera neurotrófica, em meses, foi de 101,8 meses, variando entre 10 e 360 . Quanto à localização anatômica das lesões, o Gráfico 1 demonstra que 30,0\% apresentavam-se no hálux, seguidos de região de amputação nas falanges metatarsianas e plantar, ambas com $20 \%$ de incidência. 
Gráfico 1: Distribuição anatômica das lesões neurotróficas associadas à hanseníase $(\mathrm{n}=10)$.

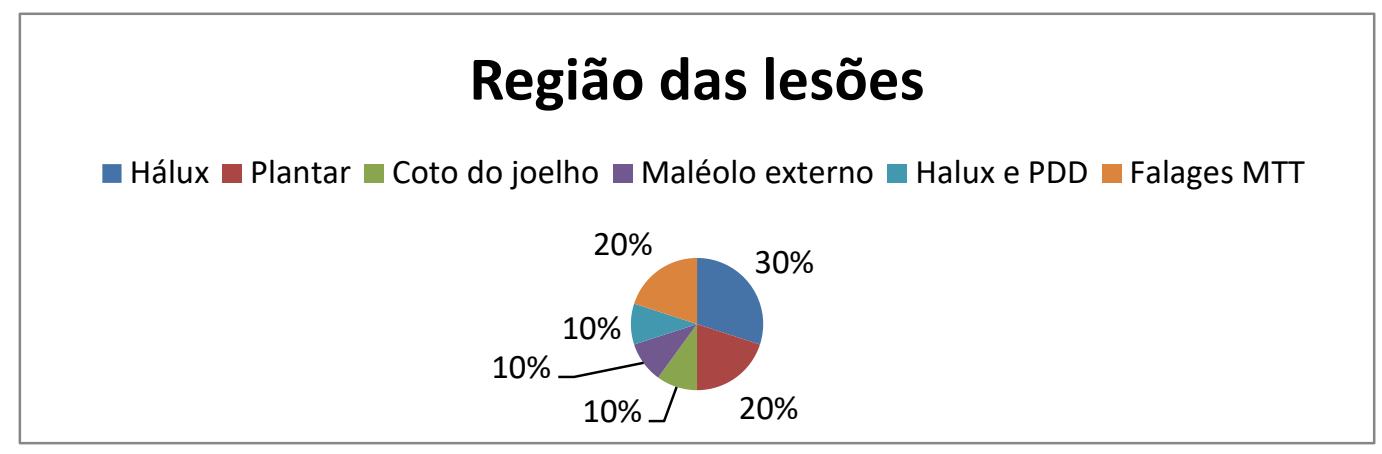

Fonte: Autores (2021).

\section{Discussão}

A hanseníase é uma infecção crônica completamente curável, então a importância em se diagnosticar precocemente, bem como de tratá-la adequadamente, detectar os prejuízos funcionais incipientes, estabelecer um plano de intervenções visando à prevenção de incapacidade e da educação dos pacientes sobre a doença está bem estabelecida na literatura. A presença de úlceras neurotróficas, como consequência, onera aos gastos públicos, o que aumenta a importância dos estudos visando tecnologias que favoreçam o processo de prevenção das incapacidades e de suas complicações (Dias et al., 2013; Chacon, 2014; Brasil, 2017; Chagas, 2018).

A média de idade foi de 44,4 \pm 11,7 anos, variando de 35 a 68 anos de idade, coincidindo com o que é referido em outros estudos, sendo associada à fase economicamente ativa da vida dos indivíduos, sob risco de exclusão da cadeia produtiva por conta de incapacidades, como consequência de uma provável demora no diagnóstico, e ressaltando a hanseníase com influência direta sobre a saúde pública (Batista et al., 2011; Lustosa et al., 2011; Santos et al., 2012).

Em face dos resultados obtidos, o perfil sociodemográfico aponta que a predominância de lesões neurotróficas foi mais expressiva no sexo masculino (70\%), este achado vai de encontro com a literatura que o explica pela possibilidade de uma maior exposição aos fatores desencadeantes da doença e ao maior cuidado das mulheres quanto ao exame dermatológico e às consultas de rotina (Pieri et al., 2012; Santana et al., 2017).

A amostra deste estudo foi caracterizada, quanto ao estado civil por maioria solteira (50\%), em contrapartida, os achados na literatura apresentam estudos onde a maior parte da amostra é composta por indivíduos que moram com companheiros, expressando a dependência do indivíduo acometido por úlcera neurotrófica para desenvolver algumas atividades da vida diária (Peres, 2018).

Quanto à raça/cor, em 2015, a taxa de detecção de casos novos de hanseníase por raça na população de cor preta e parda foi superior à observada para a população geral do país, indicando alta endemicidade da doença. Neste sentido, a predominância da cor parda $(50 \%)$ encontrada neste estudo também foi evidenciada em outros, que relacionam a esta raça à forte miscigenação existente no país, principalmente na região Nordeste (Monteiro et al., 2014; Moraes \& Furtado, 2018).

Todos os participantes da pesquisa eram procedentes do estado do Piauí, subsidiando o que Araújo (2015) pontua em seu estudo, reportando Teresina, cidade onde foi desenvolvida a pesquisa, como a segunda capital mais hiperendêmica do Brasil.

Ao analisar o grau de escolaridade da amostra estudada, percebeu-se que $60 \%$ (n=6) dos sujeitos possuíam menos de oito anos de estudo formal, o que pode ter influenciado diretamente sobre o autocuidado destes indivíduos e um fator de comprometimento da educação em saúde, uma vez que dificulta a assimilação das orientações quanto ao tratamento. Com isso, 
o indivíduo pode não considerar as consequências em longo prazo que podem ser ocasionadas devido à falta do autocuidado contínuo (Miranzi et al., 2010; Queiroz, et al., 2015).

Com relação à ocupação, observou-se que mais da metade da amostra foi constituída por aposentados (60\%), semelhante ao resultado encontrado em um estudo desenvolvido por Seixas et al. (2015), constatando 56,2\% de aposentados. Outros estudos corroboram com o achado justificando que o predomínio de indivíduos aposentados pode estar relacionado com a característica incapacitante da doença, que pode levar a uma aposentadoria por invalidez (Leite \& Caldeira, 2015; Benedicto et al., 2017).

No presente estudo, pode-se observar que a hipertensão arterial sistêmica (HAS) foi a única comorbidades presente e atingia 70\% (n=7) dos participantes da pesquisa. Santos et al. (2015) em seu estudo demonstra prevalência de HAS $(52,8 \%)$ em pacientes com úlceras em MMII. Guterres e Bordin (2010) elencam a HAS como doença mais prevalente em 73,8\% dos casos ( $\mathrm{n}=14)$, associado com lesões neurotróficas também em MMII.

Gomes et al. (2007) analisaram 79 prontuários, nos anos de 2003 e 2004, de pacientes com hanseníase: 25 participantes (32\%) apresentavam ulcerações, destes $68 \%$ apresentavam úlceras em MMII (calcâneo, hálux, região plantar e perna). Os dados da presente pesquisa retratam que os indivíduos possuíam todas as lesões em MMII (n=10). Há estudos que reforçam que houve aumento na incidência nos últimos anos destas úlceras em MMII e por seu grau de comprometimento na QV, configuram em um dos problemas de saúde pública no Brasil, evidenciando que a realidade encontrada, não se restringe a essa pesquisa (Silva \& Moreira, 2011; Fadel, 2020).

Nos resultados encontrados nesse estudo, os pés caracterizaram-se como o local mais acometido por úlceras cutâneas em 80\% dos casos. Em consonância, um estudo retrospectivo de Batista et al. (2019) obteve amostra no período de 10 anos de 256 pacientes admitidos com lesão plantar em um hospital, $11 \%$ eram pacientes com lesão nos pés por neuropatia hansênica.

A literatura traz uma maior incidência de lesões nos pés por alterações biomecânicas importantes e diminuição da sensibilidade decorrente da infecção por M. leprae, concordando com os resultados obtidos neste estudo, em que as úlceras neurotróficas analisadas variavam quanto à localização anatômica no membro, apresentando a maior porcentagem no hálux (30\%), seguido de região de amputação das falanges dos MTT e plantar, ambas com 20\% (Gomes et al., 2007; Viégas, 2019).

\section{Conclusão}

A partir desse estudo pode verificar-se que a hanseníase foi prevalente nas classes economicamente menos favorecidas, a amostra foi composta por adultos, em idade ativa, predominantemente do sexo masculino que podem refletir diferenças relacionadas a capacidade de alcance dos programas executados pelos serviços de saúde, atrelados a normativas culturais. Quanto à escolaridade, conclui-se que a população estudada possui baixa escolaridade, reforçando a necessidade de educação em saúde, visando o autocuidado. Todos os participantes eram procedentes do estão do Piauí que é apresentado como um estado mantenedor da epidemia. Metade da amostra foi composta por voluntários casados, de cor/raça parda e com reanda familiar de até dois salários-mínimos, aposentados e apresentando HAS como comorbidade.

Estes achados refletem o perfil de indivíduos com diversas limitações e situações socioeconômicas desfavoráveis, que reforçam a associação entre pobreza e hanseníase gerando necessidade de capacitação contínua dos profissionais de saúde que prestam assistência aos pacientes com hanseníase, em todos os níveis de atenção à saúde.

Destaca-se ainda, a importância da pesquisa nesta área, não apenas na busca por novos dados, mas visando traduzir o que já é sabido, otimizando a prestação do cuidado dispensado ao indivíduo. Os estudos futuros poderiam avaliar a evolução do tratamento correlacionando aos tipos de apresentação da hanseníase, demonstrando se há diferença significativa para tais classificações. 


\section{Agradecimentos}

A Deus, pela dádiva de ter encontrado pessoas tão doces e competentes ao longo do caminho que contribuíram de maneira ímpar para o desenvolvimento e escrita deste trabalho. Aos pacientes e à direção do Centro Maria Imaculada pela confiança e carinho externados à equipe de pesquisadores.

\section{Referências}

Ajalla, M. E. A., Andrade, S. M. O., Tamaki, E. M., Waissmann, W., Diettrich, S. H C. \& Silva, B. A. K. (2016). The context of leprosy in Brazil-Paraguay border. Ciência da Saúde Coletiva, 21(1), 225-232.

Almeida, F. A. F. L. \& Milan, G. (2020). Diagnóstico de hanseníase em Porto Nacional/TO no período de 2013 a 2017. Scire Salutis, 10(3), 104-112.

Almeida, P. D (2016). Incapacidades físicas e vulnerabilidade individual de casos de hanseníase em municípios hiperendêmicos. Dissertação de mestrado, Programa de Pós Graduação em Saúde e Comunidade, Universidade Federal do Piauí.

Alves, E. S., Oliveira, L. B., Araújo, T. M. E., Melo, I. V. M., Araújo, R. P. S, \& Marques, L. F (2017). Perfil epidemiológico da hanseníase em um município do nordeste brasileiro: uma análise retrospectiva. Revista de Pesquisa Cuidado é Fundamental Online, 9(3), 648-652.

Araújo, O. D., Araújo, T. M. E (2017). The operational research in leprosy. Revista de Enfermagem Universidade Federal do Piauí, 6(4), 1-3.

Araújo, O. D., Ferreira, A. F., Araújo, T. M. E., Silva, L. C. L., Lopes, W. M. P. S., Neri, E. A. R., Cardoso, J. A. et al. (2020). Mortalidade relacionada à hanseníase no Estado do Piaú, Brasil: tendências temporais e padrões espaciais, 2000-2015. Caderno de Saúde Pública, 36(9), 9391-9399.

Araújo, T. M. E. (2015). Leprosy: persistent endemic disease in Brazil with hyperendemic trend in areas of Piaui. Revista de Enfermagem UFPI, 4(2),1-3.

Arruda, M. G. M., Silva, K. T., Silva, G. N. B., Silva, R. L. \& Lopes, I. M. S. (2020). Casos de Hanseníase nas regiões Brasileiras. Brazilian Journal Of Development, 6 (11), 89427-89436.

Batista ES, Campos RX, Queiroz RCG., Siqueira, S. L., Pereira, S. M., Pacheco, T. J., Pessanha, T. O. et al. (2011). Perfil sócio-demográfico e clínicoepidemiológico dos pacientes diagnosticados com hanseníase em Campos dos Goytacazes, RJ*. Revista Brasileira de Clinica Médica, 9(2), 101-6.

Batista, K. T., Monteiro, G. B., Y-Schwartzman, U. P., Roberti, A. F. S. S. A., Rosa, A. G., Correira, C. Z. \& Silva, C. F. A. (2019). Tratamento da úlcera plantar devido à hanseníase. Revista Brasileira de Cirurgia Plástica, 34(4), 497-503.

Benedicto, C. B., Marques, T., Milano, A. P., Galan, N. G. A., Nardi, S. T., Duerksen, F., Marciano, L. H. S. C. et al. (2017). Avaliação da qualidade de vida, grau de incapacidade e do desenho da figura humana em pacientes com neuropatias na hanseníase. Acta Fisiatr., 24(3), 120-126.

Brandão, S. S., Alexandre, M. M., Nunes, P. L. P., Zardeto-Sabec, G (2020). Hanseníase: um problema de saúde pública. In Revista Eletrônica Acervo Saúde, Anais do I Congresso Online em Doenças Negligenciadas e Emergentes (p. 233). Paraná.

Brasil (2017). Ministério da Saúde. Secretaria de Vigilância em Saúde. Departamento de Vigilância das Doenças Transmissíveis. Diretrizes para vigilância, atenção e eliminação da Hanseníase como problema de saúde pública: manual técnico-operacional. Brasília: Ministério da Saúde.

Brasil (2018). Ministério da Saúde. Hanseníase- Boletim epidemiológico. Secretaria de Vigilância em Saúde/ Departamento de Vigilância Epidemiológica; Programa Nacional de Controle da Hanseníase.

Campana, A. O., Padovani, C. R., Iaria, C. T., Freitas, C. B. D., De Paiva, S. A. R, \& Hossne, W. S. (2001). Investigação científica na área médica. São Paulo: Manole.

Chacon, J. M. F. (2014). Custo do tratamento conservador da úlcera por pressão estágios III e IV em pacientes hospitalizados. Tese (Doutorado), Universidade Federal de São Paulo, Programa de Pós-Graduação em Cirurgia Translacional. 115f. São Paulo.

Chagas, I. C. S., Diniz, S. G., Lyon, S., Lyon, A. C. \& Lana, F, C. F. (2019). Fatores de risco para a ocorrência das úlceras plantares decorrente da hanseníase de acordo com a árvore de decisão. Ciência do Cuidado em Saúde, 18(3).

Chagas, I. C. S. (2018). Perfil clínico e epidemiológico dos pacientes com úlceras plantares decorrente da hanseníase. Dissertação, Programa de PósGraduação em Enfermagem, Escola de Enfermagem da Universidade Federal de Minas Gerais.

Chaves, A. E. P., Medeiros, S. M. M., Lana, F. C. F., Oliveira, A. R. \& Araújo, K. M. F. A. A. (2015). Doenças negligenciadas no estado da Paraíba: características da tuberculose e hanseníase na população de idosos. In Anais Congresso Internacional de Envelhecimento Humano. Campina Grande, PB.

Costa, J. A., Araújo, O. D., Araújo, T. M. E., Valle, A. R. M. C., Costa, J. M., Neri, E. A. R. \&Veloso, R. M. D. (2016). Leprosy: Operational Indicators in a Brazilian Hyperendemic Municipality. International Archives of Medicine, 9.

Dias, L. C., Dessoy, M. A., Guido, R.V. C., Oliva, G. \& Andricopulo, A. D. (2013). Doenças tropicais negligenciadas: uma nova era de desafios e oportunidades. Química Nova, 36(10), 1552-1556.

Fadel, A. R. M. C. (2020). Caracterização do perfil epidemiológico e demográfico de pacientes com Lesões de membros inferiores: estudo de prevalência em um hospital privado de Minas Gerais. Monografia (Especialização), Universidade Federal de Belo Horizonte. 
Gomes, F. G.; Frade, M. A. C. \& Foss, N. T. (2007). Úlceras cutâneas na hanseníase: perfil clínico-epidemiológico dos pacientes. In Investigação Clínica, Epidemiológica, Laboratorial e Terapêutica, Anais Brasileiros Dermatologia, 82(5), 433- 437.

Guterres, L. B. \& Bordin R. (2010). Qualidade de vida dos portadores de úlcera em perna cadastrados nas unidades de saúde do município de Cachoeirinha$R S$. TCC (especialização), Universidade Federal do Rio Grande do Sul.

Leite S. C. C. \& Caldeira, A. P. (2015). Oficinas terapêuticas para a reabilitação psíquica de pacientes institucionalizados em decorrência da hanseníase. Ciência da Saúde Coletiva, 20(6), 1835-42.

Lustosa, A. A., Nogueira, L T., Pedrosa, J. I. S., Teles, J. B. M \& Campelo, V. (2011). The impact of leprosy on health-related quality of life. Revista Sociedade Brasileira de Medicina Tropical, 44(5), 621-6.

Miranzi, S. S. C.; Pereira, L. H. M. \& Nunes, A. A. (2010). Perfil epidemiológico da hanseníase em um município brasileiro no período de 2000 a 2006. Revista da Sociedade Brasileira de Medicina Tropical, 43(1), 62-67.

Monteiro, L. D., Alencar, C. H., Barbosa, J. C., Novaes, C. C. B. S., Silva, R. C. P. \& Heukelbach, J. (2014). Pós-alta de hanseníase: limitação de atividade e participação social em área hiperendêmica do Norte do Brasil. Revista Brasileira de Epidemiologia, 17(1), 91-104.

Morais, J. R. \& Furtado, É. Z. L. Grau de incapacidade física de pacientes com hanseníase. Revista de Enfermagem UFPE online, $12(6)$, $1625-32$.

OMS (2016). Organização Mundial de Saúde. Biblioteca da OMS/SEARO - Estratégia mundial de eliminação da lepra 2016-2020: Acelerar a ação para um mundo sem lepra.

Oliveira, J. M. (2014). Relato de experiência sobre o uso do laser de baixa potência no tratamento de úlceras neurotróficas. Revista de Enfermagem UFPE online, 8(5), 1330-6.

Pêgo, A. F., Eleutério, D., Procópio, J. P. M., Condé, V. A. S. \& Gonçalves, E. (2020). Hanseníase: correlação entre o número de lesões hansênicas, nervos afetados e o diagnóstico precoce no estado de Minas Gerais. Revista Eletrônica Acervo Saúde / Electronic Journal Collection Health, 12(9), 1-9.

Peres, G. A. (2018). Qualidade de vida e autoestima de pessoas com feridas crônicas. Dissertação, Programa de Pós-Graduação Stricto sensu- Atenção à Saúde, Universidade Federal do Triângulo Mineiro.

Pieri, F. M., Ramos, A. C. V., Crispim, J. A., Pitiá, A. C. A., Rodrigues, L. B. B., Silveira, T. R. S. \&, Arcêncio, R. A. (2012). Fatores associados às incapacidades em pacientes diagnosticados de hanseníase: um estudo transversal. Hansenologia Internationali, 37(12), 22-30.

Queiroz, T. A., Queiroz, T; A., Carvalho, F. P. B., Simpson, C; A., Fernandes, A. C. L., \& Figueirêdo, M. I. K. (2015). Perfil clínico e epidemiológico de pacientes em reação hansênica. Revista Gaúcha de Enfermagem, 36, 185-91.

Ribeiro, M. D. A., Silva, J. C. A. \& Oliveira, S. B. Estudo epidemiológico da hanseníase no Brasil: reflexão sobre as metas de eliminação. Revista Panamericana de Salud Publica, 42.

Santana, E. M. F., Antas, E. M. V., Brito, K. K. G. \& Silva, M. A. (2017). Perfil dos pacientes acometidos pela hanseníase em um centro de atenção secundária à saúde. Revista de Enfermagem UFPE online, 11(11), 4404-9.

Santo, L. R. E., Teles, L. F., Medeiros, I. A., Silveira, M. F. \& Cordeiro, S. S. (2012). Perfil epidemiológico da hanseníase no município de brasileiro no período de 2005 a 2009. Motricidade, 8(2), 212-219.

Santos, M. D., Franco, S., Sanches, F. L. F., Rosalva, R., Hack, J., Meotti, N. \& Magro, M. (2015). Caracterização Nutricional de Pacientes com Úlceras Crônicas de Membros Inferiores em Tratamento no Ambulatório de Feridas do Campus Cedeteg da UNICENTRO, Guarapuava-PR. Cientro de Ciências Biológicas da Saúde, 17(1), p. 13-9.

Schneider, P. B. \& Freitas, B. H. B. M. Tendência da hanseníase em menores de 15 anos no Brasil, 2001-2016. Revista Caderno de Saúde Pública, 34(3), 10117.

Seixas, M. B., Loures, L. F. \& Mármora, C. H. C. (2015). Perfil sociodemográfico e clínico dos pacientes em atendimento fisioterapêutico no Hospital Universitário da Universidade Federal de Juiz. Hospital Universitário Revista, 41(2).

Silva, F. A. A. \& Moreira, T. M. M. (2011). Características sociodemográficas e clínicas de clientes com úlcera venosa de perna. Revista de Enfermagem da UERJ, 19(3), 468-72.

Viegas, M. C. (2019). Úlcera de perna: um estudo sobre registro de diagnóstico e intervenção de enfermagem em prontuário. Dissertação (Mestrado), Universidade Federal do estado do Rio de Janeiro. 